\title{
Are CP Violating Effects in the Standard Model Really Tiny?
}

\author{
A. Hernandez ${ }^{\mathrm{a}, *}$, T. Konstandin ${ }^{\mathrm{b}, * *}$, M. G. Schmidt ${ }^{\mathrm{a}, * * *}$ \\ ${ }^{a}$ Institut für Theoretische Physik, Heidelberg University, Germany \\ ${ }^{\mathrm{b}}$ Institut de Física d'Altes Energies, Universitat Autònoma de Barcelona, Spain
}

\begin{abstract}
We derive an effective action of the bosonic sector of the Standard Model by integrating out the fermionic degrees of freedom in the worldline approach. The CP violation due to the complex phase in the CKM matrix gives rise to CP-violating operators in the effective action. We calculate the prefactor of the appropriate next-to-leading order operators and give general estimates of $\mathrm{CP}$ violation in the bosonic sector of the Standard Model. In particular, we show that the effective $\mathrm{CP}$ violation for weak gauge fields is not suppressed by the Yukawa couplings of the light quarks and is much larger than the bound given by the Jarlskog determinant.
\end{abstract}

\section{Motivation and Introduction}

CP-violating effects in the Standard Model stem from the Yukawa couplings of the quarks, and are generally very small. They are due to the special flavor structure of the Standard Model [12]. To be explicit, the $\mathrm{CP}$ violation arises from the following terms in the Lagrangian

$$
Y_{i j}^{u} \bar{Q}_{L}^{i} u_{R}^{j} \phi+Y_{i j}^{d} \bar{Q}_{L}^{i} d_{R}^{j} \tilde{\phi}+\text { h.c. },
$$

where $Q_{L}$ denotes the left-handed quark $S U(2)_{L}$ doublet, $d_{R}$ and $u_{R}$ denote the righthanded quark singlets and $\phi$ denotes the Higgs doublet. We also defined the field $\tilde{\phi}$ by

$$
\tilde{\phi}=\epsilon \phi^{*}=\left(\begin{array}{cc}
0 & -1 \\
1 & 0
\end{array}\right)\left(\begin{array}{c}
\phi^{0} \\
\phi^{+}
\end{array}\right)^{*}=\left(\begin{array}{c}
-\phi^{-} \\
\phi^{0 *}
\end{array}\right),
$$

\footnotetext{
* A.Hernandez@thphys.uni-heidelberg.de

**Konstand@ifae.es

***M.G.Schmidt@thphys.uni-heidelberg.de
} 
and $Y^{u}$ and $Y^{d}$ denote the Yukawa coupling matrices. Under CP conjugation, the Yukawa couplings transform as

$$
\mathcal{C P} Y^{u / d} \mathcal{C P}^{-1}=\left(Y^{u / d}\right)^{*}
$$

such that imaginary entries in $Y^{u / d}$ potentially constitute CP violation. Spontaneous breakdown of the $S U(2)_{L}$ symmetry gives then rise to the SM quark masses. However, not all entries in the Yukawa matrices are observable. The Yukawa couplings are the only terms in the SM Lagrangian that are sensitive to global $S U(3)_{R}$ flavor transformations. This leads to the conclusion that physical observables can only depend on the combinations $m_{u} m_{u}^{\dagger}$ and $m_{d} m_{d}^{\dagger}$. In addition, there are six global phases in the left-handed quark sector that are unobservable in the SM.

In Ref. [3] it was shown that in perturbation theory the first CP odd combination of the Yukawa couplings that is invariant under these transformations is the so-called Jarlskog determinant

$$
\delta_{C P}=\operatorname{Im} \operatorname{Det}\left[m_{u} m_{u}^{\dagger}, m_{d} m_{d}^{\dagger}\right]=J \prod_{i<j} \frac{\tilde{m}_{u, i}^{2}-\tilde{m}_{u, j}^{2}}{v^{2}} \prod_{i<j} \frac{\tilde{m}_{d, i}^{2}-\tilde{m}_{d, j}^{2}}{v^{2}} \simeq 10^{-19}
$$

where $J=s_{1}^{2} s_{2} s_{3} c_{1} c_{2} c_{3} \sin (\delta)=(3.0 \pm 0.3) \times 10^{-5}$, and $\tilde{m}_{u / d}^{2}$ denotes the diagonalized mass matrices according to

$$
m_{d} m_{d}^{\dagger}=D \tilde{m}_{d}^{2} D^{\dagger}, \quad m_{u} m_{u}^{\dagger}=U \tilde{m}_{u}^{2} U^{\dagger}
$$

The Jarlskog determinant in Eq. (44) reflects the fact that CP violation is absent if any two up masses or any two down masses are equal. This is required since in this case there is an additional global flavor symmetry that can be used to remove all complex phases from the Yukawa matrices (in the SM case of three quark families). However, the above argument is based on the assumption that the observable under consideration is perturbative in the Yukawa couplings.

A baryogenesis mechanism that is based on the SM would be most compelling, but this requires that the Jarlskog determinant as an upper bound on CP violation is evaded. In principle, there are several possibilities to avoid this dilemma and to obtain a significant source of CP violaton in the SM as required by baryogenesis. For example, during a firstorder phase transition, the Higgs vev changes and hence makes it possible to construct rephasing invariants that do not only contain the masses but also their derivatives that are non-vanishing during the phase transition [4.

The possibility that concerns us here is dealing with the complicated non-perturbative dynamics by integrating out the fermions and constructing an effective action of the bosonic variables only. CP violation then appears in higher-dimensional terms of an effective Lagrangian. Main motivation for this approach is the scenario of cold electroweak baryogenesis [56] that specifically utilizes lattice simulations of the bosonic sector of the SM with higher dimensional operators that violate the CP symmetry.

\section{Effective Action}

We are interested then in the following one-loop effective action

$$
-W[\Phi, \Pi, A, B, K]=\log \operatorname{Det}\left[\not p-i \Phi(x)-\gamma_{5} \Pi(x)-\not A(x)-\gamma_{5} \not B(x)\right] .
$$


The solution is obtained for outer fields with a general internal group structure, e.g. a flavour matrix structure. Only later do we specialize to the SM. We analyze the real and imaginary parts of the effective action separately, and we are interested in the imaginary part that contains the $\mathrm{CP}$-violating contributions to the action

$$
-W^{+}-i W^{-}=\log (|\operatorname{Det}[\mathcal{O}]|)+i \arg (\operatorname{Det}[\mathcal{O}]) .
$$

To obtain the effective action we work with a worldline representation of the chiral current for which a manifestly chiral covariant expression exists. This current can then be integrated to obtain the effective action [7/89. This integration rather proceeds by matching: First, a general effective action is proposed, which has the expected chiral and covariant properties. The functional variation of this action is then matched to the covariant current that is obtained using the worldline formalism. This method has the advantage that it is both gauge and chiral invariant at each stage of the calculation. The anomaly only leads to additional complications in the matching procedure of the lowest order contributions.

As shown elegantly in Ref. [10, the imaginary part of the action can be reformulated in terms of variables that have a well-defined behaviour under chiral transformations, namely

$$
\begin{gathered}
\mathcal{A}_{\mu}=\left(\begin{array}{cc}
A_{\mu}^{L} & 0 \\
0 & A_{\mu}^{R}
\end{array}\right)=\left(\begin{array}{cc}
A_{\mu}+B_{\mu} & 0 \\
0 & A_{\mu}-B_{\mu}
\end{array}\right) \\
\mathcal{H}=\left(\begin{array}{cc}
0 & i H \\
-i H^{\dagger} & 0
\end{array}\right)=\left(\begin{array}{cc}
0 & i \Phi+\Pi \\
-i \Phi+\Pi & 0
\end{array}\right) .
\end{gathered}
$$

Next, the derivative expansion of the heat kernel is used. In the derivative expansion terms are classified by the number of covariant indices that they carry, so that $\mathcal{D}_{\mu} \mathcal{H}$ is of first order, while $\mathcal{F}_{\mu \nu}$ is of second order. The worldline formalism is well suited for this expansion for the following reasons: first, we can avoid $\gamma$-matrix algebra; second, the momentum integration is omitted and replaced by the rather trivial integration in $\tau$ space; third, the result is expressed in $x$-space; and last the method is easily implemented with computer algebra.

\section{NLO Results}

The effective action is most compactly presented in the labeled operator notation that was introduced in Ref. 9], and used also in Ref. 7]. In this notation, mass matrices obtain an additional subscript that indicates the position of the mass matrix in a subsequent product of operators. For example, using this notation we write

$$
m_{1} m_{2}^{3} m_{3}^{2} \mathcal{D}_{\mu} \mathcal{H} \mathcal{D}_{\nu} \mathcal{H}=m \mathcal{D}_{\mu} \mathcal{H} m^{3} \mathcal{D}_{\nu} \mathcal{H} m^{2} .
$$

A detailed definition and applications of this notation can be found in Ref. [9] and we refer the reader to this work.

The covariant effective action in 4-dimensions and to leading order in the covariant derivative expansion looks like

$$
W_{c}^{-}=\epsilon^{\mu \nu \lambda \sigma}\left\langle N_{123} \mathcal{D}_{\mu} \mathcal{H} \mathcal{D}_{\nu} \mathcal{H} \mathcal{F}_{\lambda \sigma}+N_{1234} \mathcal{D}_{\mu} \mathcal{H} \mathcal{D}_{\nu} \mathcal{H} \mathcal{D}_{\lambda} \mathcal{H} \mathcal{D}_{\sigma} \mathcal{H}\right\rangle
$$


It is clear that there can be no CP-violating contribution from this expression [6] and we must then look for contributions at next-to-leading order.

Using the method developed in Ref. 7] we calculated the effective action explicitly in next-to-leading order [8. The explicit form is not shown here for space concerns. Interestingly, there is only one contribution to the $\mathrm{CP}$-violating part of the effective action, namely

$$
\frac{1}{8(4 \pi)^{2}} \frac{3}{16} \frac{J \kappa^{C P}}{\tilde{m}_{c}^{2}} \epsilon^{\mu \nu \lambda \sigma} \int d^{4} x\left(Z_{\mu} W_{\nu \lambda}^{+} W_{\alpha}^{-}\left(W_{\sigma}^{+} W_{\alpha}^{-}+W_{\alpha}^{+} W_{\sigma}^{-}\right)+\text {c.c. }\right),
$$

with

$$
\kappa^{C P} \approx 9.87 \text {. }
$$

Finally, notice that the action can always be rewritten in $S U(2)_{L}$ gauge invariant quantities. For example, the charged gauge fields can be rewritten as

$$
W_{\mu \nu}^{+}=\frac{\phi^{\dagger} W_{\mu \nu} \tilde{\phi}}{\phi^{\dagger} \phi}, \quad W_{\mu \nu}^{-}=\frac{\tilde{\phi}^{\dagger} W_{\mu \nu} \phi}{\phi^{\dagger} \phi}, \quad W_{\mu}^{+}=\frac{\phi^{\dagger} \mathcal{D}_{\mu} \tilde{\phi}}{\phi^{\dagger} \phi}, \quad W_{\mu}^{-}=\frac{\tilde{\phi}^{\dagger} \mathcal{D}_{\mu} \phi}{\phi^{\dagger} \phi} .
$$

\section{Conclusions}

We calculated the CP-violating contributions to the effective action in the bosonized Standard Model in next-to-leading order in the gradient expansion. The resulting action should be valid at least for bosonic fields whose energy scale does not exceed much the charm mass. This observation is based on the fact that the action after IR regularization remains finite in the limit of vanishing up and down quark masses. We find that the coefficients of the resulting dimension-six operators are suppressed by the charm mass and the Jarlskog invariant $J$ but are many orders larger than the Jarlskog determinant $\delta_{C P}$.

Acknowledgments

T.K. is supported by the EU FP6 Marie Curie Research \& Training Network 'UniverseNet' (MRTN-CT-2006-035863). A.H. is supported by CONACYT/DAAD, Contract No. A/05/12566.

\section{References}

[1] M. Kobayashi, and T. Maskawa, Prog. Theor. Phys. 49 (1973) 652.

[2] C. Jarlskog, Z. Phys. C 29 (1985) 491.

[3] C. Jarlskog, Phys. Rev. Lett. 55 (1985) 1039.

[4] T. Konstandin, T. Prokopec and M. G. Schmidt, Nucl. Phys. B 679 (2004) 246 arXiv:hep-ph/0309291.

[5] A. Tranberg and J. Smit, JHEP 0311 (2003) 016 arXiv:hep-ph/0310342.

[6] J. Smit, JHEP 0409 (2004) 067 arXiv:hep-ph/0407161.

[7] A. Hernandez, T. Konstandin and M. G. Schmidt, Nucl. Phys. B 793 (2008) 425 arXiv:0708.0759.

[8] A. Hernandez, T. Konstandin and M. G. Schmidt, arXiv:0810.4092. 
[9] L. L. Salcedo, Eur. Phys. J. C 20 (2001) 147 arXiv:hep-th/0012166.

L. L. Salcedo, Eur. Phys. J. C 20 (2001) 161 arXiv:hep-th/0012174.

[10] E. D'Hoker and D. G. Gagne, Nucl. Phys. B 467 (1996) 297 arXiv:hep-th/9512080]. 